\title{
Modelling of three long-periodic magnetic CP-stars: HD 2453, HD 12288, and HD 200311
}

\author{
Yurij V. Glagolevskij ${ }^{1}$ and Ewald Gerth ${ }^{2}$ \\ ${ }^{1}$ Special Astrophysical Observatory, Nizhnij Arkhyz, Karachaevo-Cherkesia, 369167 Russia. \\ email: glagol@sao.ru \\ ${ }^{2}$ D-14471 Potsdam, Gontardstr. 130, Germany \\ email: ewald-gerth@t-online.de
}

\begin{abstract}
Using observational data published as phase curves of the effective magnetic field strength $B_{\mathrm{e}}(P)$ and the surface field $B_{\mathrm{s}}(P)$, magnetic models of three stars with long rotational periods are calculated by the Magnetic Charge Distribution method. For two of these stars (HD 2453 and HD 12288), the structure of the magnetic field can be described well by a central dipole model. The third star (HD 200311) is better fitted by a model of a displaced dipole, being decentered by $\triangle r=0.08 R$ along the dipole axis.
\end{abstract}

Keywords. Stars: chemical peculiar stars, stars: magnetic field, stars: individual (HD 2453, HD 12288, HD 200311)

\section{Introduction}

Models of the magnetic field of the stars HD 2453 (GK And), HD 12288 (V540 Cas), and HD 200311 (V2200 Cyg), possessing long periods of rotation, are constructed using the modelling method of the "magnetic charges distribution" (MCD-method). Owing to a duality of the solution, two variants of models are considered, first, if the obliqueness angle $\beta$ between the axes of rotation and dipole is small, and second, if $\beta$ is large. Our choice of solution always has been made in favor of the best fit to the observational data by least squares optimization.

The calculation of the magnetic field structure on the surface of a star is based on a theorem of the potential theory, according to which every vector field is determined by its sources and vortices. For the description of a static potential field the sources are alone sufficient. Vortices occur only in the case of hydrodynamical movement (e.g., the stellar magnetic dynamo). The theory underlying the MCD-method of magnetic modelling is outlined by the authors (Gerth et al. 1997, Gerth \& Glagolevskij 2000, 2001, 2004) and applied utilizing a comprehensive computer program, providing the calculation and graphical representation of the magnetic map and the phase curves of the integral magnetic field in different modes.

By means of the code for the distribution of the magnetic field in a sphere penetrated by a vector field, we calculated Mercator maps of the magnetic field distribution over the surfaces of the stars. The observational data were taken from publications of other authors (Landstreet et al. 2000, Mathys et al. 1997, Wade et al. 1997, 2000).

\section{HD 2453}

The long-periodic A1p SrCrEu-star HD 2453 with ephemeris $J D=2442288+521 E \mathrm{~d}$ (Mathys et al. , 1997) exhibits a magnetic field, which varies between $-300 \mathrm{G}$ and -1000 

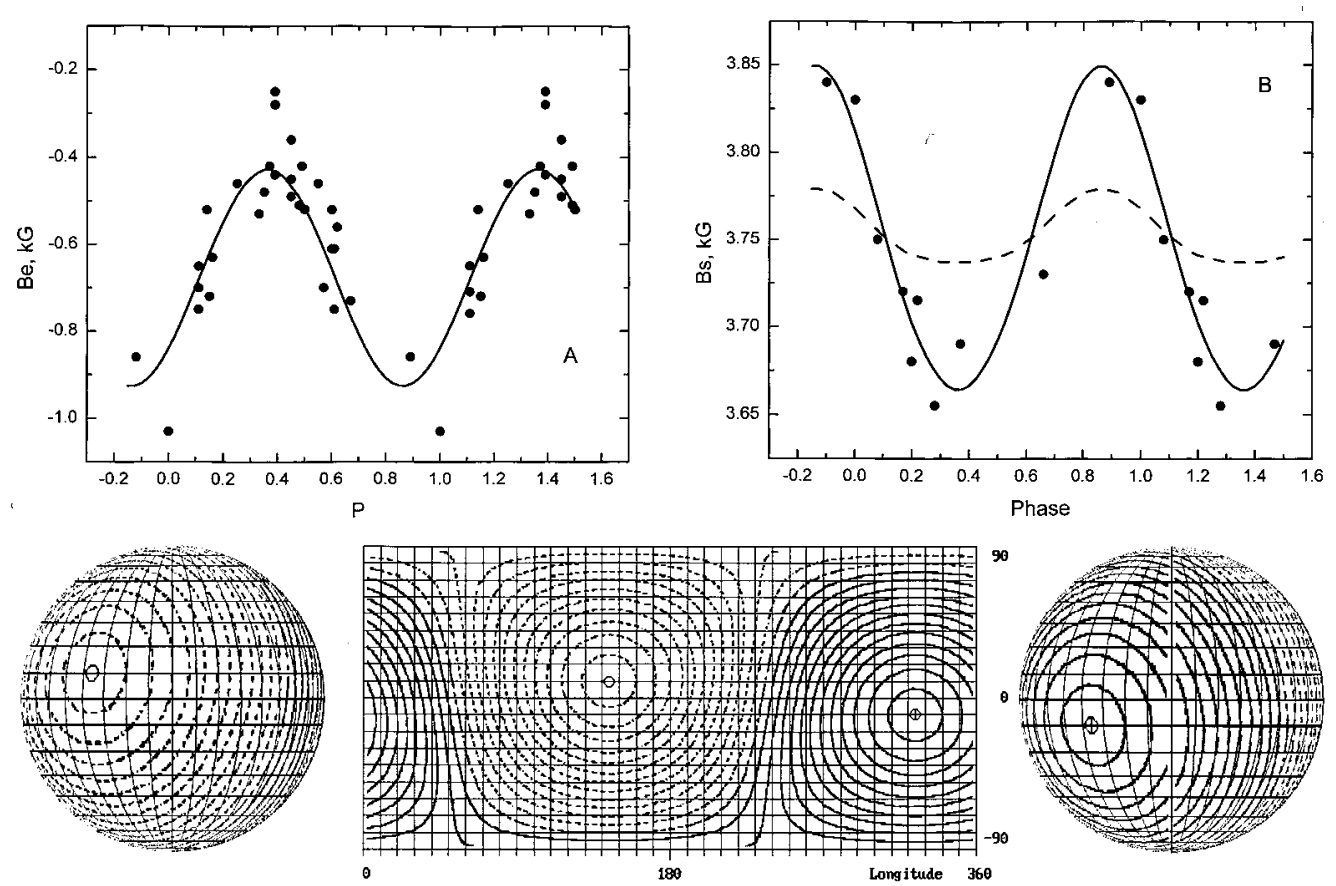

Figure 1.

Top: Phase curves of $B_{\mathrm{e}}$ and $B_{\mathrm{s}}$ of HD 2453. Dots: Observations from Wade et al. $(1997,2000)$ and Mathys et al. (1997). Solid lines - averaged from observational data.

Left panel: $B_{\mathrm{e}}$. Right panel: $B_{\mathrm{s}}$. Dotted line - calculated.

Bottom: Magnetic Mercator map - coordinates: longitude $\varphi$ and latitude $\delta$.

Globes - viewed at phases $P=0.5$ and $P=1$. Solid isomagnetic lines - region of positive polarity; dotted lines - region of negative polarity.

Derived inclination angle: $i=14^{\circ}$. Obliqueness angle: $\beta=80^{\circ}$.

Parameters (Central magnetic dipole with two separated magnetic charges $Q_{1}$ and $Q_{2}$. $B_{p}$ field strength at poles. $Q=\pi r^{2} B_{p}$. Unit star radius $R=1$ ):

\begin{tabular}{ccccc} 
Radius-fraction & Longitude & Latitude & Charge & $B_{p}[\mathrm{G}]$ \\
$r_{1}=0.1$ & $\varphi_{1}=144^{\circ}$ & $\delta_{1}=+10^{\circ}$ & $Q_{1}=-202$ & -6560 \\
$r_{2}=0.1$ & $\varphi_{2}=324^{\circ}$ & $\delta_{2}=-10^{\circ}$ & $Q_{2}=+202$ & +6560 \\
\hline
\end{tabular}

$\mathrm{G}$ without change of polarity. Thus, it could be assumed, that we look nearly pole-on at the hemisphere containing both the rotation and the negative magnetic poles. However, the reduction of the obliqueness angle $\beta$ between the rotation and the magnetic axes is ambiguous because of the inversion procedure. We have to cope with the duality of two possible solutions, which satisfy the observation. The problem is discussed and outlined in more detail by Glagolevskij \& Gerth (2004).

The couple of the angles $\beta$ and $i$ seem to have a complementary property: Small angles for $\beta$ demand large angles for $i$ and vice versa. Favoring the central dipole and presuming the approximate alignment of the axes, we model the star with a small angle of sight $i$ $=14^{\circ}$ and a large obliqueness angle $\beta=80^{\circ}$, as shown in Fig. 1 . The magnetic poles are then close to the star's equator. 

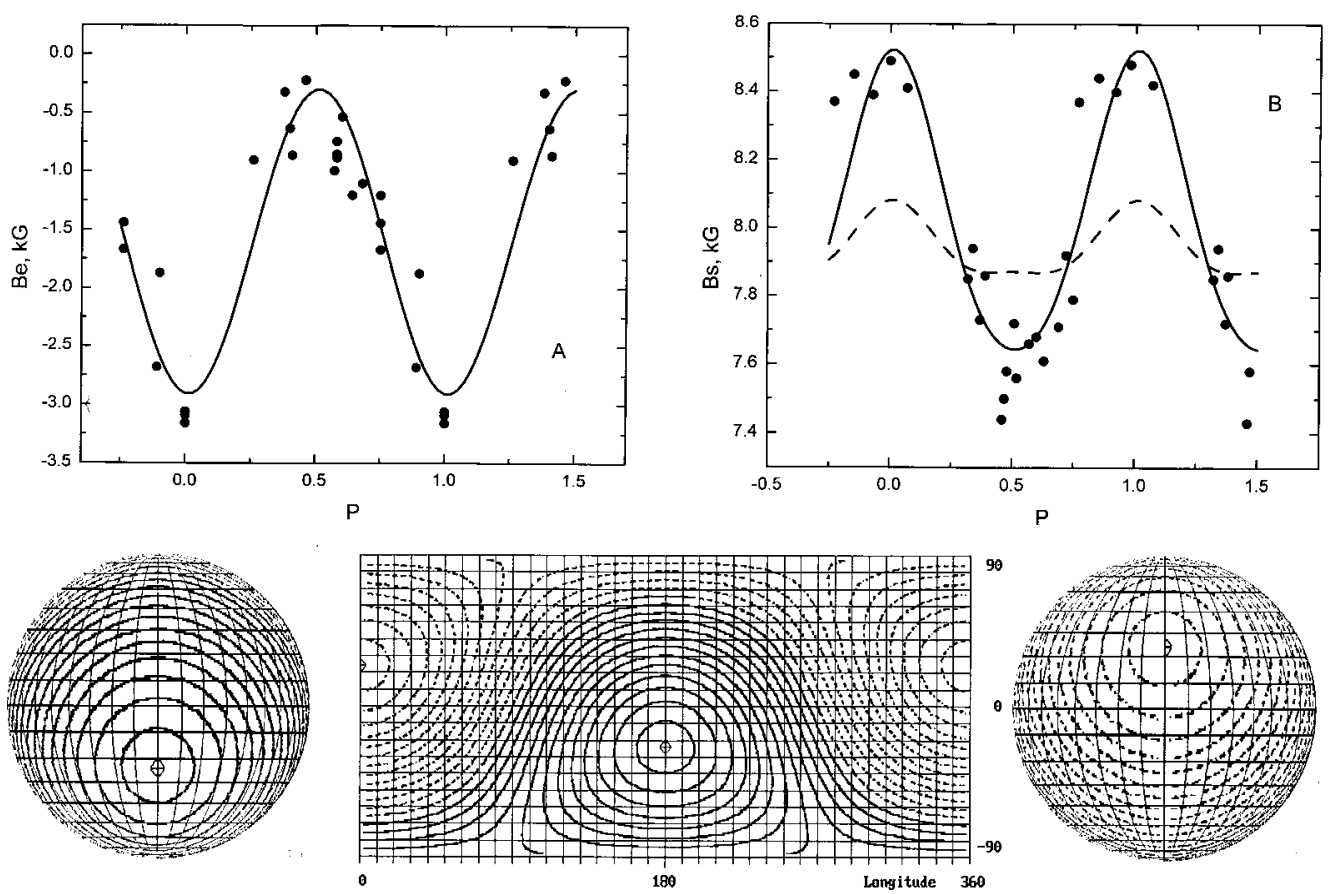

Figure 2.

Top: Phase curves of $B_{\mathrm{e}}$ and $B_{\mathrm{s}}$ of HD 12288. Observations from Wade et al. $(1997,2000)$ and Mathys et al. (1997). Bottom: Mercator map and globes.

Concerning the designations - see Fig. 1.

Derived inclination angle: $i=23^{\circ}$. Obliqueness angle: $\beta=66^{\circ}$.

Parameters (Central magnetic dipole):

$$
\begin{array}{clrcc}
\text { Radius-fraction } & \text { Longitude } & \text { Latitude } & \text { Charge } & B_{p}[\mathrm{G}] \\
r_{1}=0.1 & \varphi_{1}=0^{\circ} & \delta_{1}=+24^{\circ} & Q_{1}=-413 & -13400 \\
r_{2}=0.1 & \varphi_{2}=180^{\circ} & \delta_{2}=-24^{\circ} & Q_{2}=+413 & +13400
\end{array}
$$

\section{HD 12288}

The integrated magnetic field of the A2p SiCr-star HD 12288 varies in strength between $-300 \mathrm{G}$ and $-3000 \mathrm{G}$. Similar to HD 2453, it does not change its polarity and is observed nearly rotation pole-on. The fitting of the observational data from Wade et al. (2000) and Mathys et al. (1997) with the ephemeris given by Wade et al. $(2000) \mathrm{JD}=2448499.87+$ $34.9 \mathrm{E} \mathrm{d}$ to a model of a central dipole yields the angles $i=23^{\circ}$ and $\beta=66^{\circ}$. For this star the magnetic poles are somewhat more distant from the equator than for HD 2453. The phase curves of $B_{\mathrm{e}}$ and $B_{\mathrm{s}}$ as well as the Mercator map are shown in Fig. 2.

\section{HD 200311}

Contrary to the models of HD 2453 and HD 12288, for the SiCrHg-star HD 200311 with ephemeris $2449000+51.75 E$ (Mathys et al. 1997), the observational data are not fit sufficiently well by a central dipole model. Thus we resorted to a decentered dipole. Then, the radius fractions $r$ and the magnetic field strengths at the poles $B_{\mathrm{p}}$ are different (Fig. 3). It should be emphasized that the modelling of a decentered dipole in arbitrary position inside (or even outside) the star is the special capability of the MCD-method. 

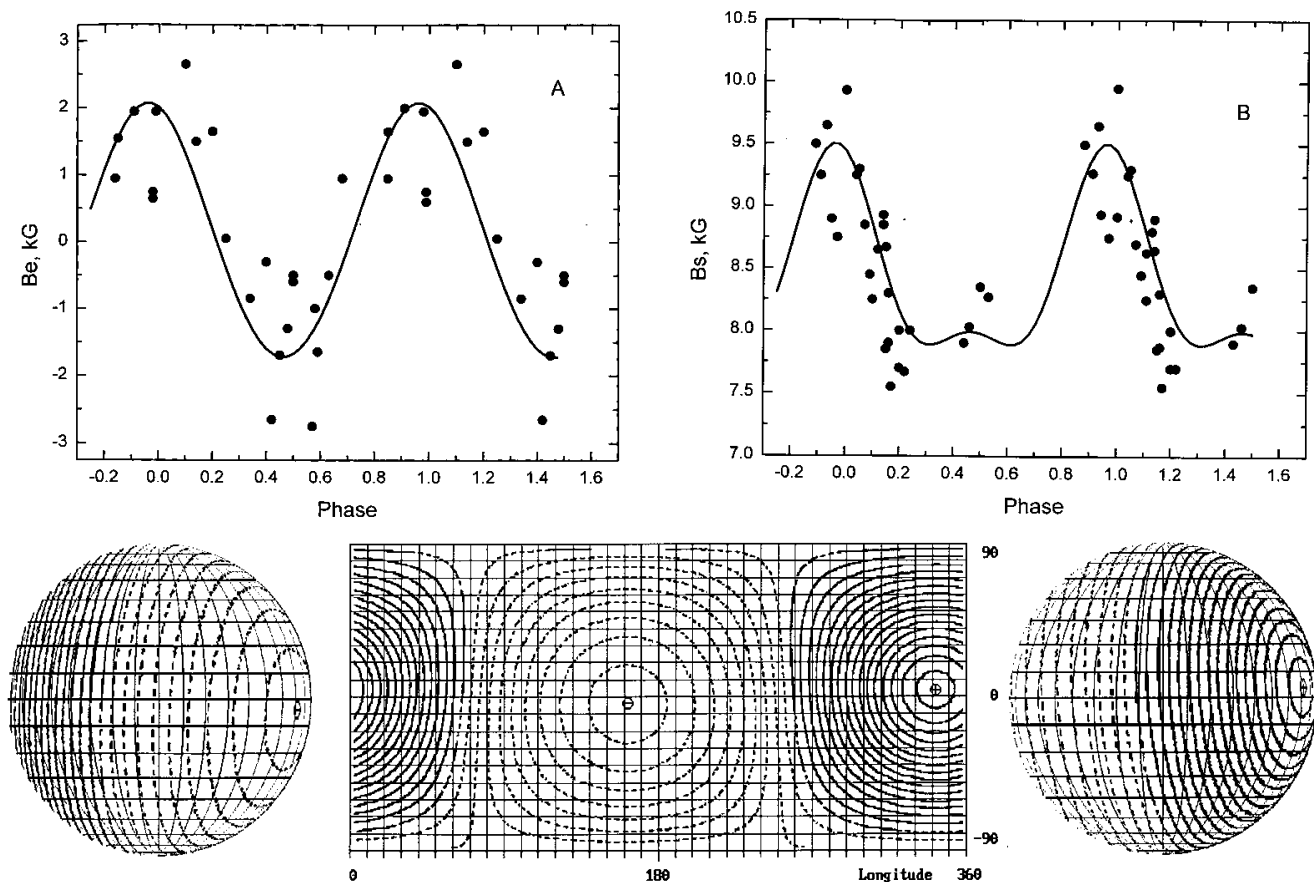

Figure 3.

Top: Phase curves of $B_{\mathrm{e}}$ and $B_{\mathrm{s}}$ of HD 200311. Observations from Wade et al. $(1997,2000)$ and Mathys et al. (1997). Bottom: Mercator map and globes.

Concerning the designations - see Fig. 1.

Derived inclination angle: $i=30^{\circ}$. Obliqueness angle: $\beta=86^{\circ}$.

Parameters (decentered magnetic dipole - displaced by $\triangle r=0.8$ ):

$\begin{array}{ccccr}\text { Radius-fraction } & \text { Longitude } & \text { Latitude } & \text { Charge } & B_{p}[\mathrm{G}] \\ r_{1}=0.18 & \varphi_{1}=342^{\circ} & \delta_{1}=+4^{\circ} & Q_{1}=+449 & 18520 \\ r_{2}=0.02 & \varphi_{2}=162^{\circ} & \delta_{2}=-4^{\circ} & Q_{2}=-449 & -11420\end{array}$

\section{Conclusion}

The periods $P$, obliqueness $\beta$ of the magnetic dipole to the rotation axis, inclination angle $i$, and absolute field strengths $B_{\mathrm{p}}$ and $B_{\mathrm{s}}$ at the magnetic poles for the stars studied are summarized in the following table:

\begin{tabular}{|c|c|c|c|c|c|c|}
\hline Star & Type & $P[\mathrm{~d}]$ & $i$ & $\beta$ & $B_{\mathrm{p}}[\mathrm{G}]$ & $B_{\mathrm{s}}[\mathrm{G}]$ \\
\hline HD 2453 & $\mathrm{~A} 1 \mathrm{p} \mathrm{SrCrEu}$ & 521 & $14^{\circ}$ & $80^{\circ}$ & \pm 6560 & 3750 \\
\hline HD 12288 & $\mathrm{~A} 2 \mathrm{p} \mathrm{SiCr}$ & 34.79 & $24^{\circ}$ & $66^{\circ}$ & \pm 13400 & 8000 \\
\hline HD 200311 & B9p SiCrHg & 51.75 & $30^{\circ}$ & $86^{\circ}$ & $\begin{array}{l}+18520 \\
-11420\end{array}$ & $\begin{array}{l}9500 \\
8100\end{array}$ \\
\hline
\end{tabular}

The structure of a bipolar magnetic surface field can be fitted to the observations and described well by a model of two oppositely charged magnetic field sources, which constitute a magnetic dipole in the star's interior. When the first attempt of modelling the field on the basis of a central magnetic dipole does not suffice, then a decentered magnetic dipole should be considered. 


\section{References}

Gerth, E., Glagolevskij, Yu.V. 2000, in Stellar Magnetic Fields (eds. Yu.V. Glagolevskij and I.I. Romanyuk), Nizhnyj Arkhyz, 151

Gerth E., Glagolevskij, Yu.V 2001, in Magnetic fields across the Hertzsprung-Russell diagram, (eds. G. Mathys, S.K. Solanki, D.T. Wickramsinghe), Santiago, Chile, 248, 333

Gerth, E., Glagolevskij, Yu.V. 2004, Bull. Spec. Astrophys. Obs., 55 (in press)

Glagolevskij, Yu.V., Gerth, E. 2002, A\&A 382, 935

Landstreet, J.D., Mathys, G. 2000, A\&A 359, 213

Mathys, G., Hubrig, S., Landstreet, J.D., Lanz, T., Manfroid, J. 1997, A\&SA Suppl. 123, 353

Wade, G.A., Elkin, V.G., Landstreet, J.D., Romanyuk, I.I. 1997, MNRAS 297, 748

Wade, G.A., Kudryavtsev, D.O., Romanyuk, I.I., Landstreet, J.D., Mathys, G. 2000, A\&A 355, 1080 